\title{
On critical extremal length for the existence of holomorphic mappings of once-holed tori
}

\author{
Makoto Masumoto* \\ Dedicated to Professor Hari M Srivastava
}

"Correspondence:

masumoto@yamaguchi-u.ac.jp Department of Mathematics,

Yamaguchi University, Yamaguchi, 753-8512, Japan

\begin{abstract}
Let $\mathfrak{T}_{a}\left[Y_{0}\right]$ be the set of marked once-holed tori which allows a holomorphic mapping into a given Riemann surface $Y_{0}$ with marked handle. We compare it with the subset $\mathfrak{T}_{\infty}\left[Y_{0}\right]$ of marked once-holed tori $X$ such that there is a holomorphic mapping $f: X \rightarrow Y_{0}$ for which the cardinal numbers of $f^{-1}(p), p \in Y_{0}$, are bounded. We show that while $\mathfrak{T}_{\infty}\left[Y_{0}\right]$ is a proper subset of $\mathfrak{T}_{a}\left[Y_{0}\right]$ apart from a few exceptions, their critical extremal lengths are identical.
\end{abstract}

MSC: Primary 30F99; secondary 32H02

Keywords: Riemann surface; once-holed torus; holomorphic mapping; extremal length

\section{Introduction}

Let $R$ be a Riemann surface of positive genus. By a mark of handle of $R$ we mean an ordered pair $\chi=\{a, b\}$ of simple loops $a$ and $b$ on $R$ whose geometric intersection number $a \times b$ is equal to one. The pair $Y=(R, \chi)$ is called a Riemann surface with marked handle.

Let $Y^{\prime}=\left(R^{\prime}, \chi^{\prime}\right)$ be another Riemann surface with marked handle, where $\chi^{\prime}=\left\{a^{\prime}, b^{\prime}\right\}$. If $f: R \rightarrow R^{\prime}$ is holomorphic and maps $a$ and $b$ onto loops freely homotopic to $a^{\prime}$ and $b^{\prime}$ on $R^{\prime}$, respectively, then we say that $f$ is a holomorphic mapping of $Y$ into $Y^{\prime}$ and use the notation $f: Y \rightarrow Y^{\prime}$. If, in addition, $f: R \rightarrow R^{\prime}$ is conformal, that is, if $f: R \rightarrow R^{\prime}$ is holomorphic and injective, then $f: Y \rightarrow Y^{\prime}$ is called conformal.

A noncompact Riemann surface of genus one with exactly one boundary component is called a once-holed torus. A marked once-holed torus means a once-holed torus with marked handle. Let $\mathfrak{T}$ denote the set of marked once-holed tori, where two marked onceholed tori are identified with each other if there is a conformal mapping of one onto the other. It is a three-dimensional real analytic manifold with boundary (see $[1, \mathbb{\$} 7]$ ).

For later use, we introduce some notations. Let $\mathbb{H}$ stand for the upper half-plane: $\mathbb{H}=$ $\{z \in \mathbb{C} \mid \operatorname{Im} z>0\}$. For $\tau \in \mathbb{H}$, let $G_{\tau}$ denote the additive group generated by 1 and $\tau$. Then $T_{\tau}:=\mathbb{C} / G_{\tau}$ is a torus, that is, a compact Riemann surface of genus one. The two oriented segments $[0,1]$ and $[0, \tau]$ are projected onto simple loops $a_{\tau}$ and $b_{\tau}$ forming a mark $\chi_{\tau}$ of handle of $T_{\tau}$. Set $X_{\tau}=\left(T_{\tau}, \chi_{\tau}\right)$. For $l \in[0,1)$, we define $T_{\tau}^{(l)}=T_{\tau} \backslash \pi_{\tau}([0, l])$, where $\pi_{\tau}$ : $\mathbb{C} \rightarrow T_{\tau}$ is the natural projection. Then $T_{\tau}^{(l)}$ is a once-holed torus. We choose a mark $\chi_{\tau}^{(l)}$ of handle of $T_{\tau}^{(l)}$ so that the inclusion mapping of $T_{\tau}^{(l)}$ into $T_{\tau}$ is a conformal mapping of $X_{\tau}^{(l)}:=\left(T_{\tau}^{(l)}, \chi_{\tau}^{(l)}\right)$ into $X_{\tau}$. The correspondence $(\tau, l) \mapsto X_{\tau}^{(l)}$ defines a bijection of $\mathbb{H} \times[0,1)$ 
onto $\mathfrak{T}$ (see, for example, [2]). In other words, every marked once-holed torus is realized as a horizontal slit domain of a torus with marked handle, or a marked torus, uniquely up to conformal automorphisms of the marked torus.

Let $Y_{0}$ be a Riemann surface with marked handle. We are interested in the set $\mathfrak{T}_{a}\left[Y_{0}\right]$ of marked once-holed tori $X$ for which there is a holomorphic mapping of $X$ into $Y_{0}$. It possesses an interesting quantitative property. In [3] (see also [4]) we have established that there is a nonnegative number $\lambda_{a}\left[Y_{0}\right]$ such that

(i) if $\operatorname{Im} \tau \geqq 1 / \lambda_{a}\left[Y_{0}\right]$, then $X_{\tau}^{(l)} \notin \mathfrak{T}_{a}\left[Y_{0}\right]$ for any $l$, while

(ii) if $\operatorname{Im} \tau<1 / \lambda_{a}\left[Y_{0}\right]$, then $X_{\tau}^{(l)} \in \mathfrak{T}_{a}\left[Y_{0}\right]$ for some $l$,

where $1 / 0=+\infty$. If $Y_{0}$ is a marked torus, then $\mathfrak{T}_{a}\left[Y_{0}\right]=\mathfrak{T}$ and hence $\lambda_{a}\left[Y_{0}\right]=0$. Otherwise, $\lambda_{a}\left[Y_{0}\right]>0$ by [5, Theorem 1 and Proposition 1$]$.

In this article we compare $\mathfrak{T}_{a}\left[Y_{0}\right]$ with the set $\mathfrak{T}_{\infty}\left[Y_{0}\right]$ of marked once-holed tori $X$ such that there is a holomorphic mapping $f: X \rightarrow Y_{0}$ for which the supremum $d(f)$ of the cardinal numbers of $f^{-1}(p), p \in R_{0}$, is finite. As is shown in [3], it possesses a property similar to that of $\mathfrak{T}_{a}\left[Y_{0}\right]$ : There is a nonnegative number $\lambda_{\infty}\left[Y_{0}\right]$ such that

(i) if $\operatorname{Im} \tau \geqq 1 / \lambda_{\infty}\left[Y_{0}\right]$, then $X_{\tau}^{(l)} \notin \mathfrak{T}_{\infty}\left[Y_{0}\right]$ for any $l$, while

(ii) if $\operatorname{Im} \tau<1 / \lambda_{\infty}\left[Y_{0}\right]$, then $X_{\tau}^{(l)} \in \mathfrak{T}_{\infty}\left[Y_{0}\right]$ for some $l$.

Since

$$
\mathfrak{T}_{\infty}\left[Y_{0}\right] \subset \mathfrak{T}_{a}\left[Y_{0}\right]
$$

we have

$$
\lambda_{\infty}\left[Y_{0}\right] \geqq \lambda_{a}\left[Y_{0}\right]
$$

We first establish the following theorem.

Theorem 1 If $Y_{0}$ is not a marked torus or a marked once-holed torus, then $\mathfrak{T}_{\infty}\left[Y_{0}\right]$ is a proper subset of $\mathfrak{T}_{a}\left[Y_{0}\right]$.

Nevertheless, the sign of equality actually holds in (1).

Theorem 2 For any marked Riemann surface $Y_{0}$, the equality $\lambda_{\infty}\left[Y_{0}\right]=\lambda_{a}\left[Y_{0}\right]$ holds.

The proofs of Theorems 1 and 2 will be given in the next section.

\section{Proofs}

We begin with the proof of Theorem 1 . Let $Y_{0}=\left(R_{0}, \chi_{0}\right)$, where $\chi_{0}=\left\{a_{0}, b_{0}\right\}$, be a Riemann surface with marked handle which is not a marked torus or a marked once-holed torus. We consider the loops $a_{0}$ and $b_{0}$ as elements of the fundamental group $\pi_{1}\left(R_{0}\right)$ of $R_{0}$. Let $\tilde{R}_{0}$ be the covering Riemann surface of $R_{0}$ corresponding to the subgroup $\left\langle a_{0}, b_{0}\right\rangle$ of $\pi_{1}\left(R_{0}\right)$ generated by $a_{0}$ and $b_{0}$. Since $R_{0}$ is not a torus, $\tilde{R}_{0}$ is a once-holed torus. We choose a mark $\tilde{\chi}_{0}=\left\{\tilde{a}_{0}, \tilde{b}_{0}\right\}$ of handle of $\tilde{R}_{0}$ so that the natural projection $\pi_{0}: \tilde{R}_{0} \rightarrow R_{0}$ is a holomorphic mapping of the marked once-holed torus $\tilde{Y}_{0}:=\left(\tilde{R}_{0}, \tilde{\chi}_{0}\right)$ onto $Y_{0}$. Then $\tilde{Y}_{0}$ is an element of $\mathfrak{T}_{a}\left[Y_{0}\right]$.

Let $f$ be an arbitrary holomorphic mapping of $\tilde{Y}_{0}$ into $Y_{0}$. Since it maps $\tilde{a}_{0}$ and $\tilde{b}_{0}$ onto loops freely homotopic to $a_{0}$ and $b_{0}$, respectively, it is lifted to a holomorphic mapping $\tilde{f}$ of 
$\tilde{Y}_{0}$ into itself satisfying $\pi_{0} \circ \tilde{f}=f$. By Huber [6, Satz II] (see also Marden-Richards-Rodin [7, Theorem 5]), we infer that $\tilde{f}$ is a conformal automorphism of $\tilde{Y}_{0}$. Since $R_{0}$ is not a torus or a once-holed torus, we conclude that $d(f)=d\left(\pi_{0}\right)=\infty$ and hence $\tilde{Y}_{0} \notin \mathfrak{T}_{\infty}\left[Y_{0}\right]$. This completes the proof of Theorem 1.

For the proof of Theorem 2, we make a remark. Let $X=(T, \chi)$, where $\chi=\{a, b\}$, be a marked once-holed torus. Then the extremal length $\lambda(X)$ of the free homotopy class of $a$ is called the basic extremal length of $X$. Note that $\lambda\left(X_{\tau}^{(l)}\right)=1 / \operatorname{Im} \tau$ (see [8, Proposition 1]).

Now, take an arbitrary $\tau \in \mathbb{H}$ with $\operatorname{Im} \tau<1 / \lambda_{a}\left[Y_{0}\right]$. Then, for some $l \in[0,1)$, there is a holomorphic mapping $f$ of $X_{\tau}^{(l)}$ into $Y_{0}$. Recall that $T_{\tau}^{(l)}$ is the horizontal slit domain $T_{\tau} \backslash \pi_{\tau}([0, l])$ of the torus $T_{\tau}$. Choose a canonical exhaustion $\left\{S_{n}\right\}$ of $T_{\tau}^{(l)}$ so that each $S_{n}$ is a once-holed torus including the loops in $\chi_{\tau}^{(l)}$. Since the inclusion mapping $S_{n} \rightarrow$ $T_{\tau}^{(l)}$ is a conformal mapping of the marked once-holed torus $W_{n}:=\left(S_{n}, \chi_{\tau}^{(l)}\right)$ into $X_{\tau}^{(l)}$, the restriction $f_{n}$ of $f$ to $S_{n}$ is a holomorphic mapping of $W_{n}$ into $Y_{0}$. As $S_{n}$ is relatively compact in $T_{n}^{(l)}$, we know that $d\left(f_{n}\right)<\infty$. Consequently, $W_{n}$ belongs to $\mathfrak{T}_{\infty}\left[Y_{0}\right]$.

To estimate the basic extremal length of $W_{n}$, take an arbitrary positive number $\varepsilon$ less than $\operatorname{Im} \tau / 2$. Let $H_{\varepsilon}$ be the horizontal strip $\{z \in \mathbb{C} \mid \varepsilon<\operatorname{Im} z<\operatorname{Im} \tau-\varepsilon\}$. Since $\left\{S_{n}\right\}$ is increasing with $\bigcup_{n} S_{n}=T_{\tau}^{(l)}$, for all sufficiently large $n$, the subdomain $S_{n}$ includes the ring domain $\pi_{\tau}\left(H_{\varepsilon}\right)$. It follows that

$$
\operatorname{Im} \tau-2 \varepsilon<\frac{1}{\lambda\left(W_{n}\right)} \leqq \frac{1}{\lambda_{\infty}\left[Y_{0}\right]}
$$

which implies that

$$
\operatorname{Im} \tau \leqq \frac{1}{\lambda_{\infty}\left[Y_{0}\right]} .
$$

As $\tau$ was an arbitrary point of $\mathbb{H}$ satisfying $\operatorname{Im} \tau<1 / \lambda_{a}\left[Y_{0}\right]$, we deduce that

$$
\frac{1}{\lambda_{a}\left[Y_{0}\right]} \leqq \frac{1}{\lambda_{\infty}\left[Y_{0}\right]}
$$

or

$$
\lambda_{\infty}\left[Y_{0}\right] \leqq \lambda_{a}\left[Y_{0}\right]
$$

Theorem 2 has been proved.

\section{Topological relations between $\mathfrak{T}_{a}\left[Y_{0}\right]$ and $\mathfrak{T}_{\infty}\left[Y_{0}\right]$}

The arguments in the proof of Theorem 2 easily lead us to the following theorem.

Theorem 3 The closure of $\mathfrak{T}_{\infty}\left[Y_{0}\right]$ is identical with $\mathfrak{T}_{a}\left[Y_{0}\right]$.

Proof We begin with recalling a global coordinate system on the space $\mathfrak{T}$ of marked onceholed tori. Let $X=(T, \chi)$ be a marked once-holed torus, where $\chi=\{a, b\}$. Observe that $\dot{\chi}:=\left\{b, a^{-1}\right\}$ is a mark of handle of $T$. Also, if $c$ is a simple loop homotopic to $a b^{-1}$, then $\ddot{\chi}:=$ $\{c, a\}$ is another mark of handle of $T$. Set $\dot{X}=(T, \dot{\chi})$ and $\ddot{X}=(T, \ddot{\chi})$. Then the basic extremal lengths of $X, \dot{X}$ and $\ddot{X}$ define a global coordinate system on $\mathfrak{T}$. In fact, we introduce a real 
analytic structure into $\mathfrak{T}$ so that the mapping $\Lambda: X \mapsto(\lambda(X), \lambda(\dot{X}), \lambda(\ddot{X}))$ is a real analytic diffeomorphism of $\mathfrak{T}$ into $\mathbb{R}^{3}$ (see [1]).

Now, let $X=(T, \chi)$ be an arbitrary element of $\mathfrak{T}_{a}\left[Y_{0}\right]$. Take a canonical exhaustion $\left\{S_{n}\right\}$ of $T$ for which each $S_{n}$ is a once-holed torus including the loops in $\chi$, and set $W_{n}=\left(S_{n}, \chi\right)$. Since $X=X_{\tau}^{(l)}$ for some $\tau \in \mathbb{H}$ and $l \in[0,1)$, the proof of Theorem 2 shows that the basic extremal length $\lambda\left(W_{n}\right)$ tends to $\lambda(X)$ as $n \rightarrow \infty$. By changing marks of handles, we infer that $\left\{\lambda\left(\dot{W}_{n}\right)\right\}$ and $\left\{\lambda\left(\ddot{W}_{n}\right)\right\}$ converge to $\lambda(\dot{X})$ and $\lambda(\ddot{X})$, respectively, and hence that $\Lambda\left(W_{n}\right) \rightarrow \Lambda(X)$ as $n \rightarrow \infty$. Since each $W_{n}$ belongs to $\mathfrak{T}_{\infty}\left[Y_{0}\right]$, the marked once-holed torus $X$ belongs to the closure $\overline{\mathfrak{T}_{\infty}\left[Y_{0}\right]}$ of $\mathfrak{T}_{\infty}\left[Y_{0}\right]$. We thus obtain $\mathfrak{T}_{a}\left[Y_{0}\right] \subset \overline{\mathfrak{T}_{\infty}\left[Y_{0}\right]}$. Because $\mathfrak{T}_{a}\left[Y_{0}\right]$ is closed and includes $\mathfrak{T}_{\infty}\left[Y_{0}\right]$, we conclude that $\overline{\mathfrak{T}_{\infty}\left[Y_{0}\right]}=\mathfrak{T}_{a}\left[Y_{0}\right]$.

Since $\mathfrak{T}_{\infty}\left[Y_{0}\right]$ and $\mathfrak{T}_{a}\left[Y_{0}\right]$ are (noncompact) domains with Lipschitz boundary by [3], we see that Theorem 3 is an improvement of Theorem 2. Also, we obtain the following corollary.

Corollary 1 The interiors of $\mathfrak{T}_{\infty}\left[Y_{0}\right]$ and $\mathfrak{T}_{a}\left[Y_{0}\right]$ coincide with each other.

If $Y_{0}$ is a marked torus, then $\mathfrak{T}_{a}\left[Y_{0}\right]$ is identical with $\mathfrak{T}$ (see [1]). Hence so is $\mathfrak{T}_{\infty}\left[Y_{0}\right]$ by Corollary 1.

Competing interests

The author declares that he has no competing interests.

\section{Acknowledgements}

This research is supported in part by JSPS KAKENHI Grant Number 22540196. The author is grateful to the referees for their invaluable comments.

Received: 15 December 2012 Accepted: 22 May 2013 Published: 5 June 2013

References

1. Masumoto, M: Conformal mappings of a once-holed torus. J. Anal. Math. 66, 117-136 (1995)

2. Shiba, M: The moduli of compact continuations of an open Riemann surface of genus one. Trans. Am. Math. Soc. 301 299-311 (1987)

3. Masumoto, M: Holomorphic mappings of once-holed tori. Preprint

4. Masumoto, M: Conformal and holomorphic mappings of once-holed tori. Global J. Math. Sci. 1, 24-30 (2012)

5. Masumoto, M: Holomorphic mappings and basic extremal lengths of once-holed tori. Nonlinear Anal. 71, e1178-e1181 (2009)

6. Huber, H: Über Analytische Abbildungen Riemannscher Flächen in sich. Comment. Math. Helv. 27, 1-73 (1953)

7. Marden, A, Richards, I, Rodin, B: Analytic self-mappings of Riemann surfaces. J. Anal. Math. 18, 197-225 (1967)

8. Masumoto, M: Estimates of the Euclidean span for an open Riemann surface of genus one. Hiroshima Math. J. 14, 573-582 (1992) 